\title{
Three-dimensional color map: a novel tool to locate the surgical transepicondylar axis
}

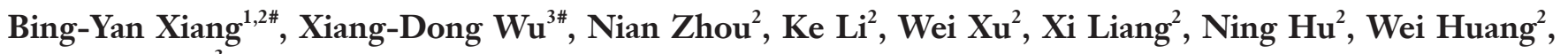 \\ Gui-Xing Qiu ${ }^{3}$
}

${ }^{1}$ Department of Orthopaedic Surgery, The Third Affiliated Hospital of Zunyi Medical University (The First People's Hospital of Zunyi), Zunyi, China; ${ }^{2}$ Department of Orthopaedic Surgery, The First Affiliated Hospital of Chongqing Medical University, Chongqing, China; ${ }^{3}$ Department of Orthopaedic Surgery, Peking Union Medical College Hospital, Chinese Academy of Medical Sciences \& Peking Union Medical College, Beijing, China

Contributions: (I) Conception and design: BY Xiang, XD Wu, W Huang; (II) Administrative support: BY Xiang, W Huang, GX Qiu; (III) Provision of study materials or patients: All authors; (IV) Collection and assembly of data: BY Xiang, XD Wu, N Zhou, K Li, W Xu, X Liang, N Hu; (V) Data analysis and interpretation: BY Xiang, XD Wu, N Zhou, K Li, W Huang, GX Qiu; (VI) Manuscript writing: All authors; (VII) Final approval of manuscript: All authors. \#These authors contributed equally to this work.

Correspondence to: Bing-Yan Xiang, MD, PhD. Department of Orthopaedic Surgery, The First Affiliated Hospital of Chongqing Medical University, No. 1, Youyi Road, Yuanjiagang, Yuzhong District, Chongqing 400016, China; Department of Orthopaedic Surgery, The Third Affiliated Hospital of Zunyi Medical University (The First People’s Hospital of Zunyi), Zunyi 563003, China. Email: 1024178414@qq.com; Wei Huang, MD, PhD. Department of Orthopaedic Surgery, The First Affiliated Hospital of Chongqing Medical University, No. 1, Youyi Road, Yuanjiagang, Yuzhong District, Chongqing 400016, China. Email: drhuangwei68@gmail.com.

Background: Accurate localization of the surgical transepicondylar axis (sTEA) in total knee arthroplasty (TKA), the most reliable anatomical reference for femoral rotation, has long been a challenge, primarily because it is intractable to locate the center of the sulcus of the medial epicondyle. This study aimed to introduce and verify a novel method to locate the sTEA more precisely.

Methods: This study included 26 adult femoral specimens and 80 adult patients with computed tomography (CT) scan data. Three dimensions (3D) models based on CT scans of the distal femurs were reconstructed with Mimics and imported into Geomagic Studio. The 3D color map method was applied to locate the sTEA. To further verify the accuracy of the method, the identified sTEA was transferred to the femoral specimens and compared with the points identified by the total station machine. We further compared the recognition rate of sTEA between 3D color map method and two-dimensional (2D) CT slices method. The repeatability of this novel method was also evaluated.

Results: The 3D color map method located the centers of the sulcus of the medial epicondyle and the most prominent point of the lateral epicondyle of all the femoral specimens, which were further identified and confirmed by patient-specific guide plates and total station machine on femoral specimens. The $3 \mathrm{D}$ color map method achieved a recognition rate of up to $96.23 \%$, while the recognition rate of the 2D CT slices method was only $68.87 \%$. The repeatability of this objective method was excellent.

Conclusions: The results of this study indicated that the 3D color map method could be used to accurately and objectively locate the sTEA, with high repeatability and recognition rate. However, the proposed novel method requires further validation in clinical applications.

Keywords: 3D color map; distal femoral rotational alignment; Geomagic Studio; transepicondylar axis; total knee arthroplasty (TKA)

Submitted Feb 24, 2020. Accepted for publication Sep 04, 2020.

doi: 10.21037/atm-20-1887

View this article at: http://dx.doi.org/10.21037/atm-20-1887 


\section{Introduction}

Rotational alignment of the distal femur is an important reference axis for successful total knee arthroplasty (TKA), but its location has long been a challenge, and anatomical references are often used to locate the rotational alignments. Anatomical references are based on the acquisition of anatomical landmarks, such as the transepicondylar line (TEA) (1-3), the posterior condylar line (PCL) (4-6), and the Whiteside's line (WL) (7-10). The intraoperative localization, function, and reliability of these landmarks for determining femoral component rotation have been extensively studied (1,4,7,11-16). The surgical transepicondylar axis (sTEA) has been recognized as the most reliable anatomical reference to determine the correct rotation of the femoral component, and it is not affected by bone geometry, such as condylar osteophyte or bone defects (17-23).

The sTEA is a line joining the center of the sulcus of the medial epicondyle and the most prominent point of the lateral epicondyle. Locating the sulcus of the medial epicondyle is particularly difficult, mainly due to concealment by the soft tissues of the medial collateral ligament that attach over the medial sulcus and medial epicondyle $(1,24,25)$. Previous studies have reported high deviation of location, low intraobserver and interobserver reliability and reproducibility, and low recognition rates of the location of the sTEA by using computed tomography (CT) or magnetic resonance imaging (MRI) (24-27), and no golden standard method is available. Therefore, a reliable method to accurately identify and digitize the sTEA is desperately needed for research and clinical application.

A three-dimensional (3D) color map is a kind of graphical representation of the distance differences between 2 superimposed 3D images. Although many software programs that are compatible with $3 \mathrm{D}$ imaging data also have the capability of generating 3D color maps, 3D color maps are rarely used in the orthopedic field, as some surgeons may be unaware of how to generate such maps and how to interpret them. This study first introduced the $3 \mathrm{D}$ color map method to enhance the accuracy of the sTEA location and aimed to validate the repeatability and recognition rate of this novel approach. We hypothesized that the 3D color map method could accurately locate the sTEA, with a higher repeatability and recognition rate than the previous two-dimensional (2D) CT slices method. We present the following article in accordance with the Materials Design Analysis Reporting (MDAR) checklist (28) (available at http://dx.doi.org/10.21037/atm-20-1887).

\section{Methods}

The study was conducted in accordance with the Declaration of Helsinki (as revised in 2013) (29). Ethical approval of this study was obtained from the Ethics Committee of The First Affiliated Hospital of Chongqing Medical University (NO.: 2016-94), and informed consent for this specific project was waived because the research was performed on radiographic data. The following experiment was conducted following the approved study protocol.

\section{Materials and population}

This study included 26 adult femur specimens (15 left femurs, 11 right femurs) and 80 adult patients (31 males, 49 females, with 41 left femurs, 39 right femurs), which were scanned with a Siemens Somatom Definition AS 128-slice CT scanner. The CT scans (slice thickness, $0.625 \mathrm{~mm}$ ) were saved as digital imaging and communications in medicine (DICOM) files. The average age of the 80 patients was 38.4 years (range from 23 to 65 years), and none of the patients presented with a history of distal femoral fractures or surgery. Specifically, femur specimens were also CT scanned and used for further verification (Figure 1).

\section{sTEA localization with the 3D color map}

3D models of the distal femurs were reconstructed with Mimics 15.0 (Materialise HQ, Leuven, Belgium) and imported into Geomagic Studio 2013 (Geomagic $^{\circledR}$, Morrisville, North Carolina). The distal femur of each subject was placed in a standard position (the medial and lateral condyles of the distal femur were superimposed) for the following procedures: (I) Create a plane: The Best Fit application was used to select the medial and lateral femoral epicondyles of each femur, and the plane command was used to identify the area and assign a name to each plane for further analysis, alignment, and trimming. (II) 3D color map: A 3D color-coded mapping was generated based on the distance differences between the plane and the underlying polygonal mesh. Next, the center of the sulcus of the medial epicondyle and the most prominent point of the lateral epicondyle were accurately located based on the colors at the positive or negative end of the scale. The sTEA, which is the connection between the two points, was identified and digitized by using the 3D color map method (Figure 2). 


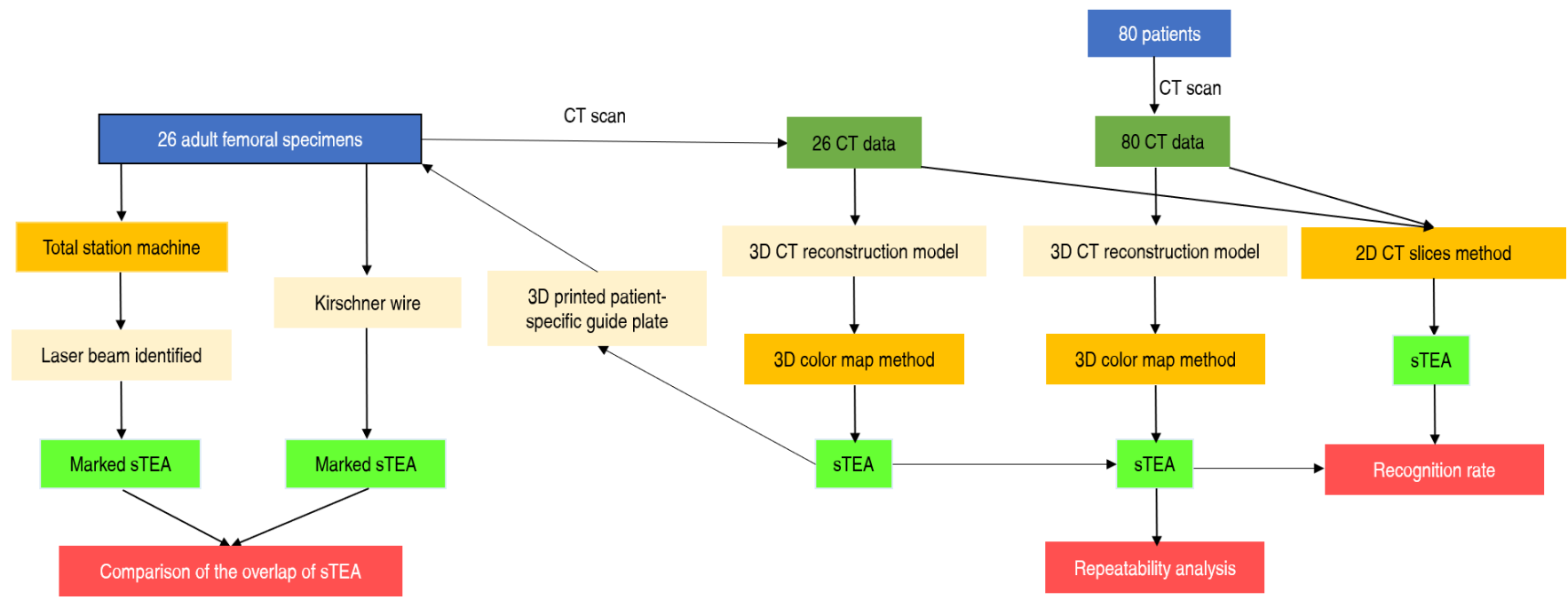

Figure 1 Flow chart of the study procedure. sTEA, surgical transepicondylar axis.
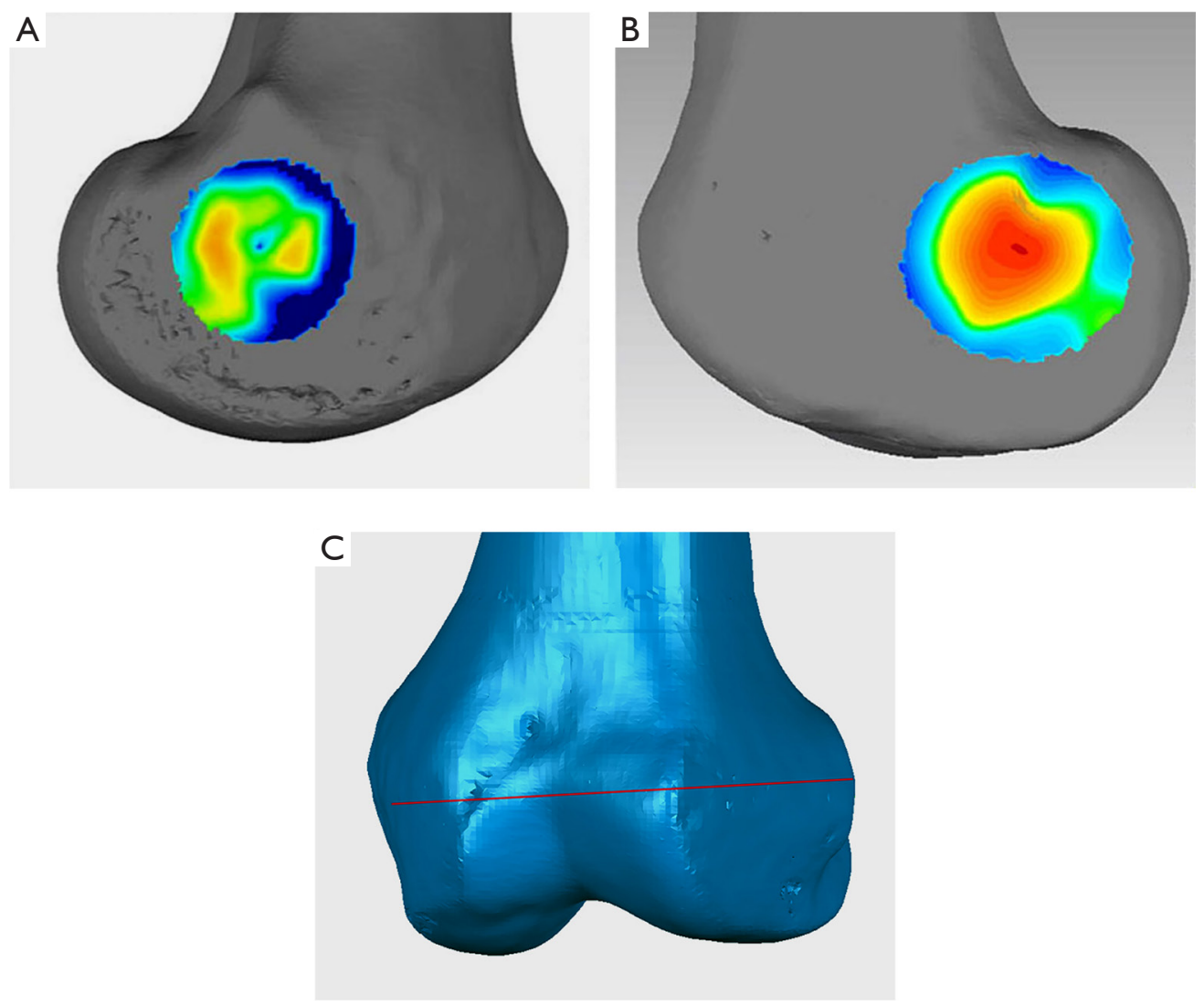

Figure 2 sTEA localization with the 3D color map method (application of Best Fit), in a standard position (the medial and lateral condyles of the distal femur were superimposed). (A) The 3D color map was used to locate the center of the sulcus of the medial epicondyle. (B) Localization of the most prominent point of the lateral epicondyle using the 3D color map. (C) Localization of the sTEA, a line between the center of the sulcus of the medial epicondyle and the most prominent point of the lateral epicondyle. 

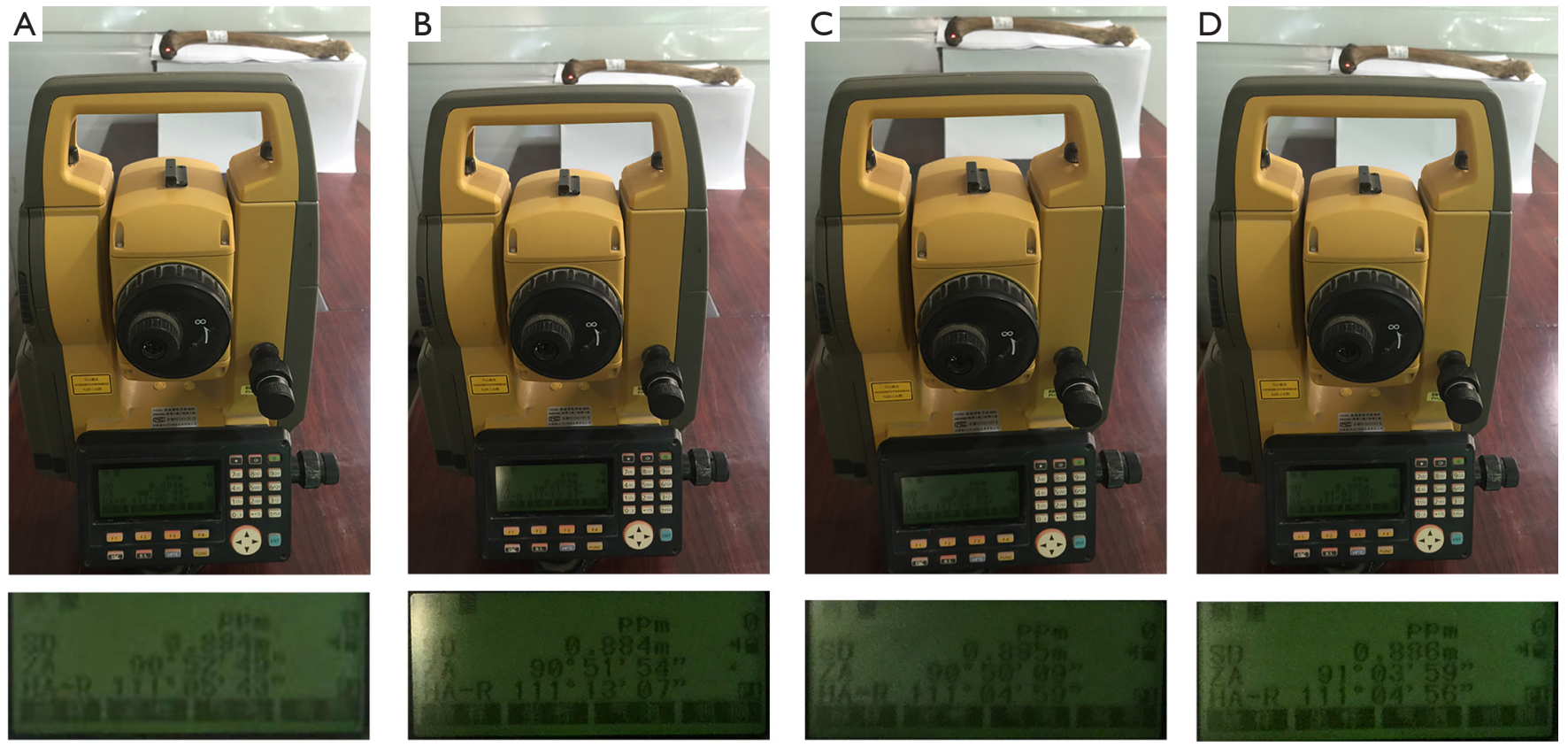

$0.884 \mathrm{~m}$

$0.884 \mathrm{~m}$

$0.885 \mathrm{~m}$

$0.886 \mathrm{~m}$

Figure 3 Verification with the total station machine. The horizontal distance between the center of the sulcus of the medial epicondyle and the most prominent point of the lateral epicondyle of each specimen were measured repeatedly. The center of the sulcus of the medial epicondyle was furthest from the total station machine, while the most prominent point of the lateral epicondyle was nearest to the total station machine.

\section{sTEA verification}

\section{sTEA identified by a total station machine}

Total stations are mainly used by land surveyors and civil engineers, either to record features as in topographic surveying or to set out features, which is a high-precision equipment through a combination of electromagnetic distance measuring instruments and electronic theodolites. A total station machine (accuracy of $\pm 0.2 \mathrm{~mm}$, Topcon ES$602 \mathrm{G}$, Beijing, China) was applied to locate the anatomical landmarks of the medial and lateral epicondyles on the femoral specimens and to locate the sTEA on specimens. According to the measurement principle, the telescope eyepiece of the total station machine and the medial or lateral femoral condyle was placed in the same horizontal plane (Figure 3). The horizontal distance between the total station machine and the medial or lateral femoral epicondyle was repeatedly measured to determine the center of the sulcus of the medial epicondyle or the most prominent point of the lateral epicondyle. The center of the sulcus of the medial epicondyle was the furthest point from the total station machine, while the most prominent point of the lateral epicondyle was the nearest point to the total station machine, which were identified by a laser beam and marked for further comparison.

\section{Verification with the patient-specific guide plate}

To evaluate the consistency between the points located by the 3D color map and the anatomical landmarks of the medial and lateral epicondyles on the femoral specimens, the location of sTEA and the geometry of the distal femur were used to design and construct a patient-specific guide plate with guide holes (diameter: $2.0 \mathrm{~mm}$ ) via $3 \mathrm{D}$ printing (Figure 4). Then, Kirschner wires ("K-wires", diameter: $1.5 \mathrm{~mm}$ ) were passed through the guide holes to locate the sTEA axes found by the $3 \mathrm{D}$ color map on the femoral specimens. The consistency of the points located by the $3 \mathrm{D}$ color map and the patient-specific guide plate was compared to the anatomical landmarks identified by the total station machine.

\section{sTEA recognition rate of the $3 D$ color map}

A previous study reported that the sulcus of the medial epicondyle, which could be used to identify the medial end of the sTEA, might become filled in as osteoarthritis 

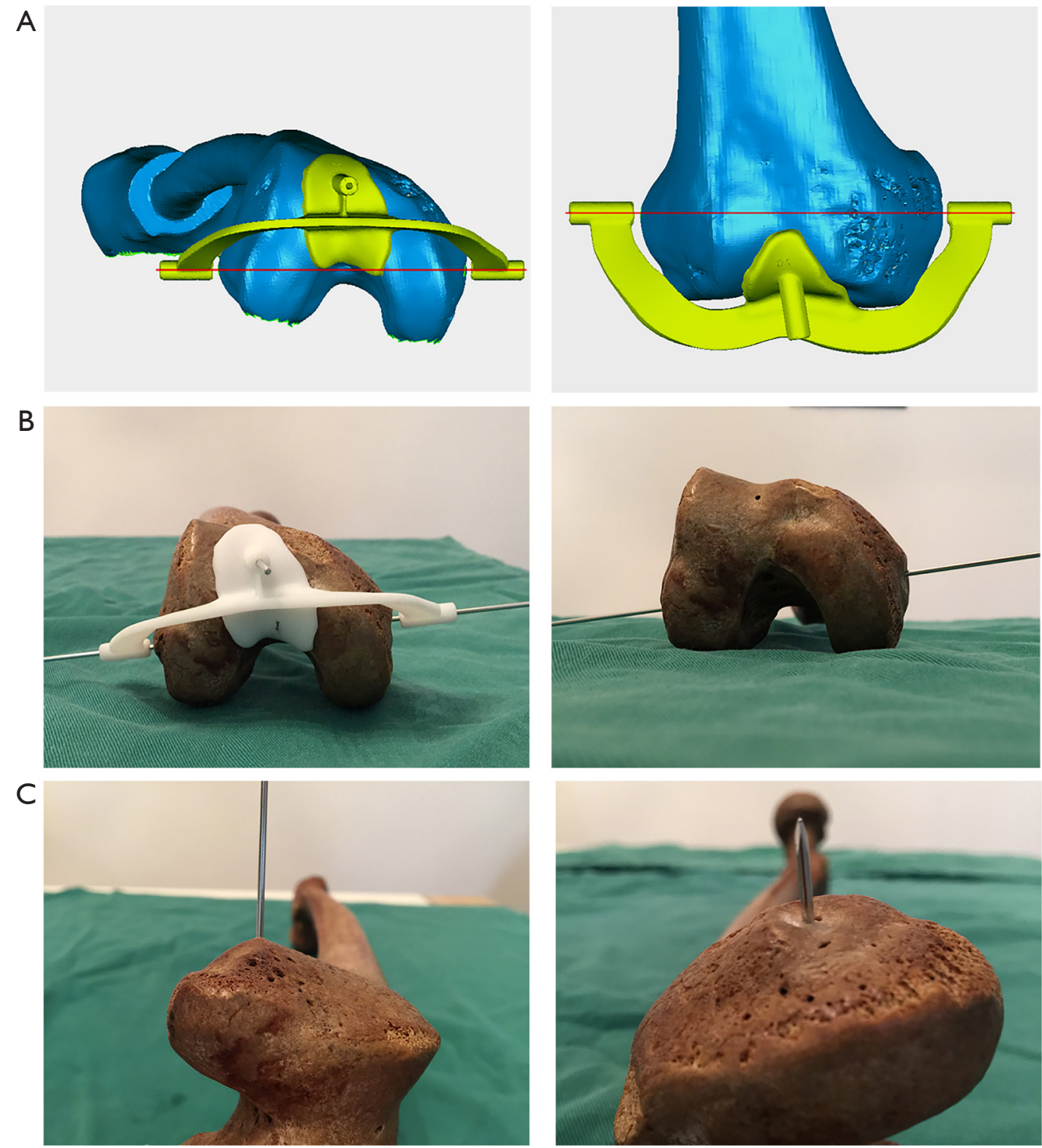

Figure 4 Verification with the patient-specific guide plate. (A) Patient-specific guide plate models with guide holes (diameter: 2.0 mm) were constructed for each femoral specimen via 3D printing based on the surgical transepicondylar axis (sTEA) localization results. (B) Kirschner wire (diameter: $1.5 \mathrm{~mm}$ ) was passed through the guide hole in each femoral specimen. (C) Comparison of the consistency of the points on medial epicondyle and lateral epicondyle of each specimen identified by using the Kirschner wire and total station machine.

progresses; thus, the sulcus may not be identified in some cases. Therefore, the recognition rate, which was defined as the proportion of located sTEA of all cases, was used to compare the 3D color map method with the 2D CT slices method. The 106 CT scans (including 26 femur specimens and 80 patients) of the femur were collected to construct 3D bone models. Each specimen was used to recognize and locate the sTEA by using the 3D color map method, and the recognition rate was calculated accordingly.

\section{Repeatability analysis of the 3D color map}

The posterior condylar angle (PCA) was chosen as the repeatability parameter. The PCA was defined as the angle between the PCL and the sTEA. Before repetitive measurements, we established a $3 \mathrm{D}$ coordinate system to ensure that all the measurements were carried out in the same spatial location. The coordinate system was defined previously (Figure 5) (30). Measurements were made three 


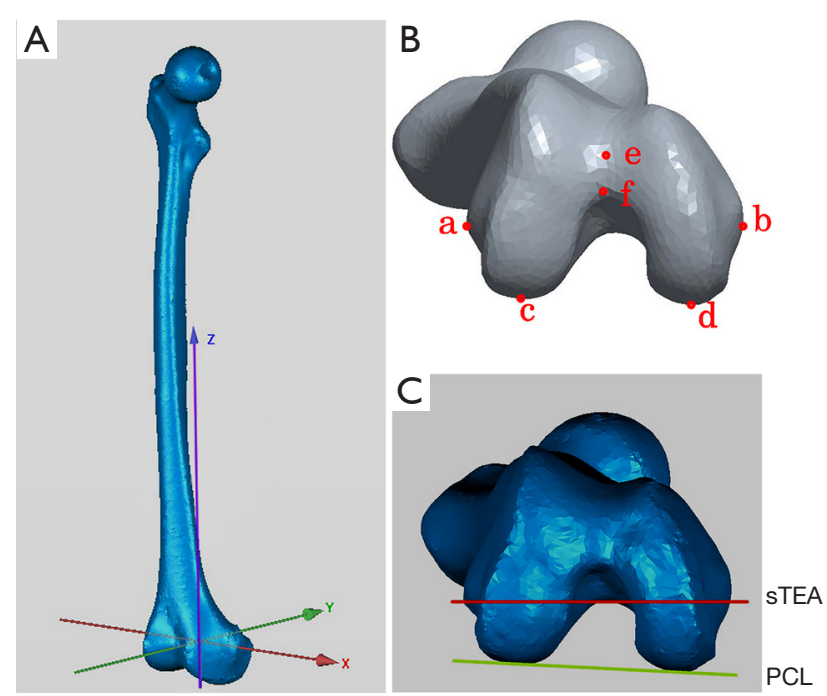

Figure 5 The posterior condylar angle (PCA) - the angle between the posterior condylar line (PCL) and the surgical transepicondylar axis (sTEA) was determined. (A) Three-dimensional coordinate system for measurement: The center of the hip was taken as a sphere fitted to the femoral head. The center of the knee was identified as the mid-point of the sTEA. The Z-axis of the knee (distal-proximal) was defined as the extension of the femoral mechanical axis that connected the center of the knee and the center of the femoral head. The plane perpendicular to the $\mathrm{Z}$-axis at the center of the knee was defined as the $\mathrm{X}-\mathrm{Y}$ plane. The $\mathrm{X}$-axis (medial-lateral) was defined as the extension of the sTEA, which was projected onto the $\mathrm{X}-\mathrm{Y}$ plane perpendicular to the $\mathrm{Z}$-axis. The $Y$-axis (anterior-posterior) was defined as the line normal to the coronal plane (X-Z plane) at the center of the knee. (B) Important anatomical landmarks identified by the $3 \mathrm{D}$ color map method. (a: The most prominent point of the lateral epicondyle; b: The center of the sulcus of the medial epicondyle; c: The lowest point of the lateral femoral condyle; $d$ : The lowest point of the medial femoral condyle; e: The top of the femoral notch; f: The deepest point of the trochlear groove). (C) PCA was the angle between PCL and sTEA lines.

times by two orthopedic surgeons and a radiologist at an interval of two weeks to assess the interobserver and intraobserver reliability of the $3 \mathrm{D}$ color map method. The CT scan data of the 80 patients were used for the repeatability analysis. The observers were not aware of any previous measurements at the time.

\section{Statistical analysis}

All of the data were analyzed using SPSS version 18.0
(SPSS Inc, Chicago, Illinois, USA). The associations among the various measurements were evaluated using Pearson's correlation coefficient. A P value $<0.05$ was considered statistically significant. The intraobserver and interobserver reliabilities were determined based on the intraclass correlation coefficients (ICCs) of each measurement (26). ICC values greater than 0.90 indicate excellent reliability, while ICC values between 0.75 and 0.9 indicate good reliability, values between 0.5 and 0.75 indicate moderate reliability, and values less than 0.5 are indicative of poor reliability.

\section{Results}

\section{sTEA localization and verification}

The center of the sulcus of the medial epicondyle and the most prominent point of the lateral epicondyle in the 26 femur specimens were all accurately located via the 3D color map approach (Figure 2). Based on the horizontal distance, the center of the sulcus of the medial epicondyle and the most prominent point of the lateral epicondyle were identified and marked on all femoral specimens by using a total station machine (Figure 3). The patient-specific guide plates and $\mathrm{K}$-wires successfully marked the center of the sulcus of the medial epicondyle and the most prominent point of the lateral epicondyle on the femoral specimens (Figure 4), which were all overlaid with the points marked by the total station machine.

\section{sTEA recognition rate of the $3 D$ color map}

The 3D color map method showed a higher recognition rate of $96.23 \%$ (102 of 106 cases) than the 2D CT slices method $68.87 \%$ (73 of 106 cases). The ages of the patients for which the method failed were $28,30,35$, and 56 years old, respectively.

For instance, in some cases, the center of the sulcus of the medial epicondyle was unable to be accurately located via 2D CT slices (Figure 6A) but could be precisely located with a 3D color map (Figure $6 B$ ). Notably, in one particular case, the center of the sulcus of the medial epicondyle could be located in eight consecutive thin-slice CT images (Figure $7 A$ ), when the eight CT slices were automatically converted into a $3 \mathrm{D}-\mathrm{CT}$ reconstruction model, which merged into a sulcus approximately $5 \mathrm{~mm}$ in length (Figure $7 B)$. However, the exact center of the sulcus was able to be precisely located by the $3 \mathrm{D}$ color map (Figure $7 C$ ). 

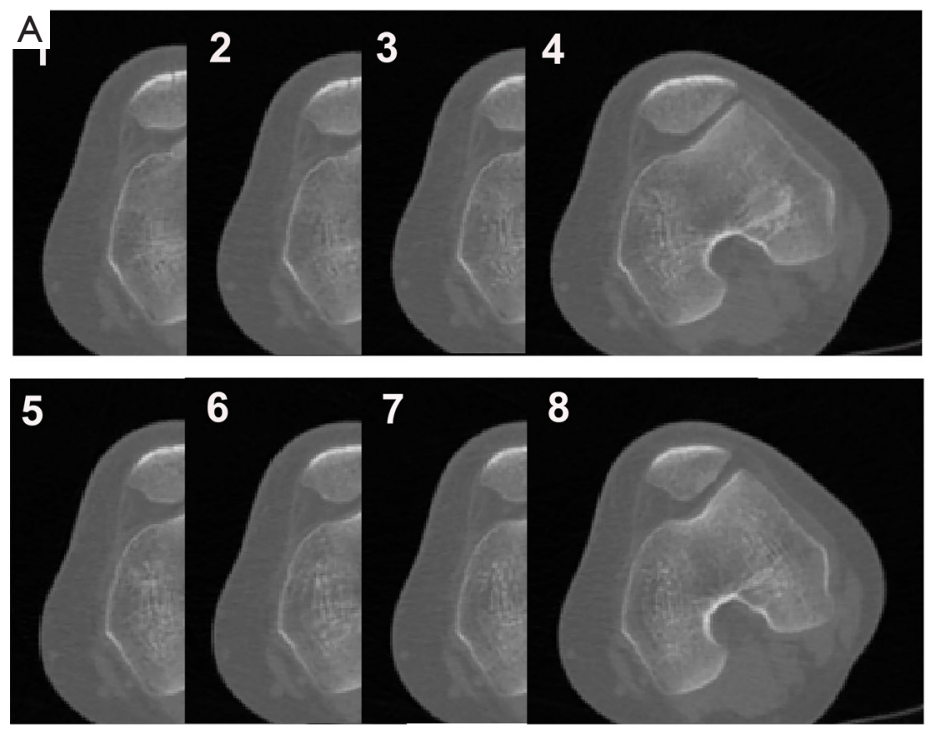

\section{B}

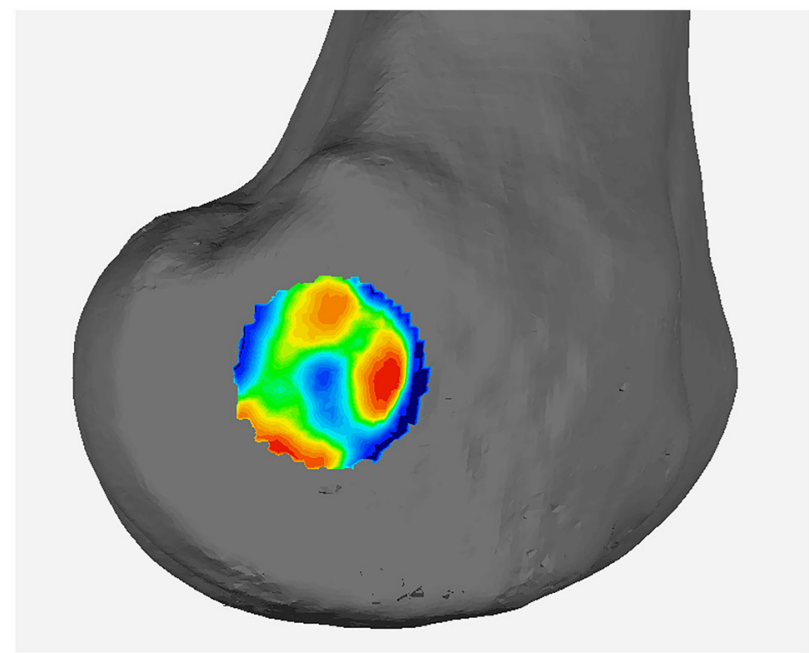

Figure 6 The center of the sulcus of the medial epicondyle was unable to be accurately located via the 2D CT slices method, but could be located via 3D color map method. (A) In this case, the center of the sulcus of the medial epicondyle was unable to be accurately located in these consecutive slices via the 2D CT slices method. (B) The center of the sulcus of the could be precisely located via the 3D color map method. (Low values are dark blue, values in the center of the map are green, and high values are dark red).

Similarly, the most prominent point on the lateral epicondyle of the femur appeared in eight consecutive thinslice CT images (Figure 7D), which merged into a ridge in the 3D-CT reconstruction model (Figure $7 E$ ), but the exact most prominent point could be precisely located using the 3D color map (Figure $7 F)$. In this case, the maximum angle of the error of the two endpoints was $7.52^{\circ}$ when the sTEA was set at $7.8 \mathrm{~cm}$ in length (Figure $7 G$ ).

In most cases, the center of the sulcus of the medial epicondyle and the most prominent point of the lateral epicondyle were located in different 2D CT slices, but the sTEA was a line in $3 \mathrm{D}$ space (Figure 8 ). Thus, the actual sTEA was often not reflected in one $2 \mathrm{D}$ thin-slice CT image.

\section{Repeatability analysis of the $3 D$ color map}

The repeatability of the $3 \mathrm{D}$ color map method was evaluated by measuring PCA (Figure $5 B, C$ ). The ICC values for intraobserver and interobserver reliability for different measurements on PCA were 0.993 and 0.985 , respectively $(\mathrm{P}<0.001)$. Meanwhile, the correlation coefficient was 0.986 for two intraobserver observations, and the correlation coefficient was 0.973 for two interobserver observations (Table 1 and Table 2). The results indicate excellent repeatability of the 3D color map method.

\section{Discussion}

\section{Main findings}

To the best of our knowledge, this is the first study that introduced the $3 \mathrm{D}$ color map method into the orthopedic field to locate the center of the sulcus of the medial epicondyle and the most prominent point of the lateral epicondyle, and to accurately and objectively locate the sTEA. The reliability and recognition rate of the proposed method were higher than those of the 2D CT slices method.

\section{Implications for clinical practice}

Although TKA is associated with greater than $90 \%$ survival for more than ten years after implantation (31-33), up to $15-30 \%$ of patients remain "unsatisfied" after TKA (34-37). The accuracy of the rotational alignment of the femoral component is considered to be one of the most influential factors of short-term functional outcome and long-term implant survival $(38,39)$. Although sTEA has been well established as the most reliable reference for the rotational axis of the distal femur, it is difficult to accurately 

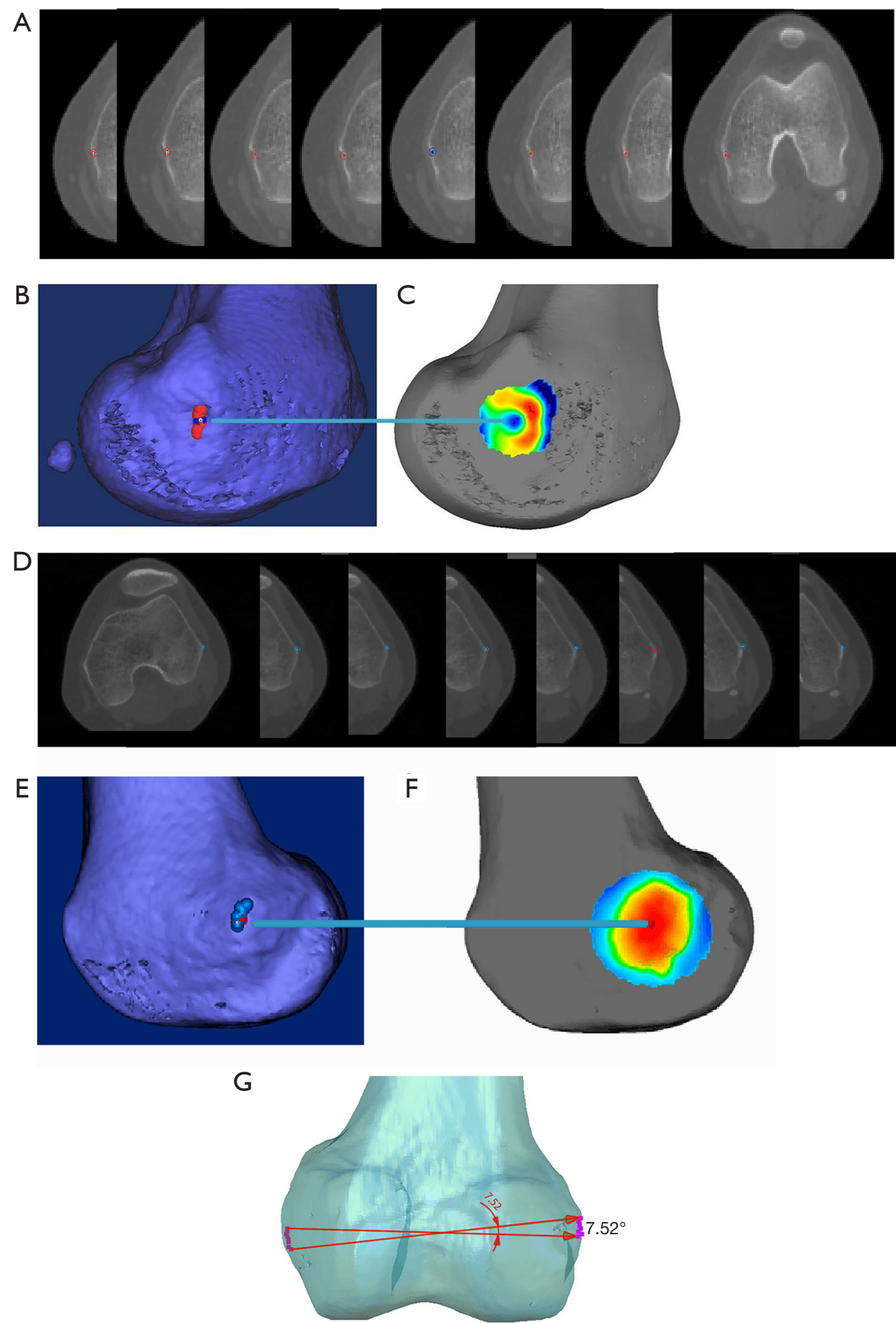

Figure 7 Comparison between the 3D color map method and 2D CT slices method. (A) The center of the sulcus of the medial epicondyle was able to be located in eight consecutive thin-slice CT images. (B) All of the points were automatically converted into the 3D reconstruction model, which merge into a sulcus approximately $5 \mathrm{~mm}$ in length. (C) The $3 \mathrm{D}$ color map method could locate the center of the sulcus of the medial epicondyle. (D) The most prominent point of the lateral epicondyle appears in eight consecutive thin-slice CT images. (E) All of the points were automatically converted into the $3 \mathrm{D}$ reconstruction model, which merged into a ridge approximately $5 \mathrm{~mm}$ in length. (F) The $3 \mathrm{D}$ color map method could locate the most prominent point of the lateral epicondyle. (G) The maximum angle of the error of the two endpoints was $7.52^{\circ}$ when the length of the sTEA was $7.8 \mathrm{~cm}$ in length (Low values are dark blue, values in the center of the map are green, and high values are dark red). 

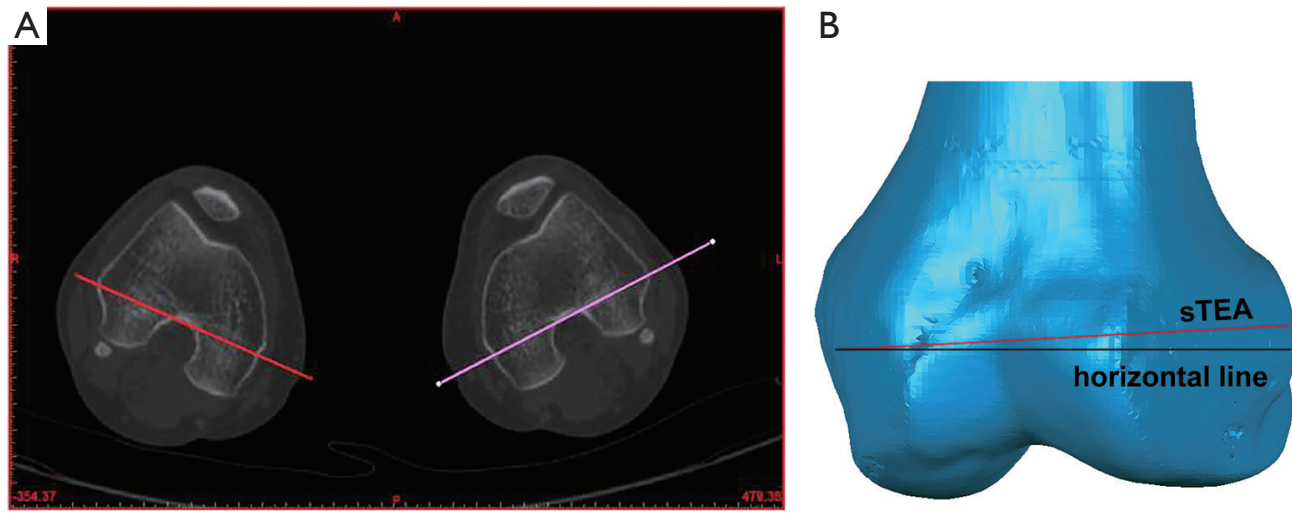

Figure 8 The sTEA located by the 3D color map method. (A) The sTEA was located in one CT slice via the 2D CT slices method. (B) The sTEA located by the $3 \mathrm{D}$ color map method was an axis in the $3 \mathrm{D}$ space.

Table 1 Intraobserver reliability on different measurements [WithinR1 (3 measures)]

\begin{tabular}{|c|c|c|c|c|c|c|}
\hline & \multicolumn{2}{|c|}{ Reliability } & \multicolumn{4}{|c|}{ Difference } \\
\hline PCA & 0.986 & 0.993 & $<0.001$ & 3.662 & 3.648 & 3.676 \\
\hline
\end{tabular}

ICC, intraclass correlation coefficient; PCA, Posterior Condylar Angle.

Table 2 Interobserver reliability for different observers [R1(mean)-R2]

\begin{tabular}{|c|c|c|c|c|c|c|}
\hline & \multicolumn{2}{|c|}{ Reliability } & \multicolumn{4}{|c|}{ Difference } \\
\hline PCA & 0.973 & 0.985 & $<0.001$ & 3.620 & 3.578 & 3.662 \\
\hline
\end{tabular}

ICC, intraclass correlation coefficient; PCA, Posterior Condylar Angle.

locate sTEA during surgery, mainly due to the medial epicondyle is covered by extensive soft tissue. Jerosch et al. suggested a high perioperative variance of sTEA for rotational alignment of the femoral component (24), which could induce inaccurate prosthesis implantation and various postoperative complications. Although PCL and WL have been created to determine the rotational alignment of the femoral component (40-42), individual differences in bone morphology, such as bone deformity or defects of the femoral condyle, and other factors including gender, race, height, and even weight, can affect the relationships between these parameters and sTEA (26,43-47). Therefore, these parameters are inaccurate and unreliable, which may result in adverse outcomes in patients after TKA.

The 2D CT slices method has been traditionally used to locate the sTEA, but proved to be inaccurate with a low recognition rate. Yoshino et al. included 48 participants but only detected the medial sulcus in 33 knees and suggested that the degree of osteoarthritis significantly affected the identification of the medial sulcus (25). Our study also revealed that the $2 \mathrm{D}$ CT slices method yielded a low recognition rate of sTEA, and the center of the sulcus of the medial epicondyle was only recognized in 73 of 106 patients $(68.87 \%)$. In contrast, the $3 \mathrm{D}$ color map method showed a high recognition rate, only 4 of 106 cases failed, which was not due to apparent osteoarthritis but due to abnormal morphology of the medial epicondyle. Therefore, the $3 \mathrm{D}$ color map method could be used to precisely and objectively locate the sTEA in most cases, but may also fail in some cases with abnormal femur morphology.

Our findings demonstrated that the 2D CT slices method is inaccurate for sTEA localization, mainly due 
to the following reasons: (I) The center of the sulcus of the medial epicondyle and the most prominent point of the lateral epicondyle could be found in consecutive thinslice CT images, which merged into a sulcus in the medial epicondyle or a ridge in the lateral epicondyle in the $3 \mathrm{D}$ CT reconstruction model. Even if these marked points were automatically traced in the $3 \mathrm{D}$ reconstruction model, it would be highly challenging to locate the correct point objectively. (II) The center of the sulcus of the medial epicondyle and the most prominent point of the lateral epicondyle were often not located in the same slice from the CT scans. (III) Recognition of the center of the sulcus of the medial epicondyle and the most prominent point of the lateral epicondyle was often based on the surgeon's experience and subjective evaluations. Based on the inaccurate location of anatomical landmarks of the distal femur, other crucial parameters, including the PCA, the Whiteside-epicondylar angle (W-EP angle), and the condylar twist angle (CTA), would be unreliable. In comparison, the $3 \mathrm{D}$ color map method is an objective method, with high repeatability and reliability.

\section{Call for future studies}

This study indicated that the 3D color map method could be used to precisely and objectively locate the center of the sulcus of the medial epicondyle, the most prominent point of the lateral epicondyle, and the sTEA. These recognized points can be transferred from the $3 \mathrm{D}-\mathrm{CT}$ model to femoral specimens with a $3 \mathrm{D}$-printed patient-specific guide plate, which holds great promise for clinical applications, especially for improving the design of patient-specific instrumentation (PSI) in TKA in the future (48). Therefore, this study established a novel method for future research regarding the rotation axis of the distal femur. In addition, the $3 \mathrm{D}$ color map method could be used to digitize and explore the morphological features of the distal femur and to locate other important anatomical landmarks, especially the PCA, W-EP angle, and CTA. The color map method can also be applied in other $3 \mathrm{D}$ geometric morphometric analyses.

\section{Limitations}

This study had some limitations. First and foremost, there is no golden standard method for locating the sTEA; and we were unable to compare the accuracy of the $3 \mathrm{D}$ color map method with the 2D CT slices method, mainly due to different models. Second, the Kellgren-Lawrence (KL) classification of the included patients was not available; therefore, it remains uncertain whether the sTEA can be accurately located in patients with severe knee osteoarthritis by using the $3 \mathrm{D}$ color map method, even though the degenerative processes of osteoarthritis only involves damage to the articular surface and is associated with marginal osteophytes. Next, CT scans are associated with high levels of radiation exposure. Finally, the proposed method has not yet been applied in clinical applications and requires further validation.

\section{Conclusions}

The findings of this study suggest that the $3 \mathrm{D}$ color map method could be used to precisely locate the sTEA, with high repeatability and recognition rate. The proposed approach could be used to locate the sTEA and other important anatomical landmarks. However, the proposed method requires further validation in clinical applications.

\section{Acknowledgments}

Funding: This study was supported by a grant from Special Fund for Scientific and Technological Cooperation of Zunyi, Guizhou (2019-178 to Bing-Yan Xiang) and grants from the Natural Science Foundation of China (81572142 and 81371972 to Wei Huang). Funders of this study had no role in the study design, data collection and analysis, interpretation of results, preparation of the manuscript or decision to publish the manuscript.

\section{Footnote}

Reporting Checklist: The authors have completed the Materials Design Analysis Reporting (MDAR) checklist. Available at http://dx.doi.org/10.21037/atm-20-1887

Data Sharing Statement: Available at http://dx.doi. org/10.21037/atm-20-1887

Conflicts of Interest: All authors have completed the ICMJE uniform disclosure form (available at http://dx.doi. org/10.21037/atm-20-1887). The authors have no conflicts of interest.

Ethical Statement: The authors are accountable for all aspects of the work in ensuring that questions related 
to the accuracy or integrity of any part of the work are appropriately investigated and resolved. The study was conducted in accordance with the Declaration of Helsinki (as revised in 2013). The ethical approval of this study was obtained from the Ethics Committee of The First Affiliated Hospital of Chongqing Medical University (NO.: 2016-94), and informed consent for this specific project was waived because the research was performed on radiographic data, which cannot be used to identify personal information.

Open Access Statement: This is an Open Access article distributed in accordance with the Creative Commons Attribution-NonCommercial-NoDerivs 4.0 International License (CC BY-NC-ND 4.0), which permits the noncommercial replication and distribution of the article with the strict proviso that no changes or edits are made and the original work is properly cited (including links to both the formal publication through the relevant DOI and the license). See: https://creativecommons.org/licenses/by-nc-nd/4.0/.

\section{References}

1. Berger RA, Rubash HE, Seel MJ, et al. Determining the rotational alignment of the femoral component in total knee arthroplasty using the epicondylar axis. Clin Orthop Relat Res 1993:40-7.

2. Park A, Duncan ST, Nunley RM, et al. Relationship of the posterior femoral axis of the "kinematically aligned" total knee arthroplasty to the posterior condylar, transepicondylar, and anteroposterior femoral axes. Knee 2014;21:1120-3.

3. Pedraza W, Beckmann J, Mayer C, et al. Partially loaded plain radiographic measurement to evaluate rotational alignment in total knee arthroplasty. Int Orthop 2016;40:2519-26.

4. Yau WP, Chiu K, Tang W. How precise is the determination of rotational alignment of the femoral prosthesis in total knee arthroplasty: an in vivo study. J Arthroplasty 2007;22:1042-8.

5. Lee DH, Park JH, Song DI, et al. Accuracy of soft tissue balancing in TKA: comparison between navigation-assisted gap balancing and conventional measured resection. Knee Surg Sports Traumatol Arthrosc 2010;18:381-7.

6. Moon YW, Seo JG, Lim SJ, et al. Variability in femoral component rotation reference axes measured during navigation-assisted total knee arthroplasty using gap technique. J Arthroplasty 2010;25:238-43.

7. Middleton FR, Palmer SH. How accurate is Whiteside's line as a reference axis in total knee arthroplasty? Knee 2007;14:204-7.

8. Hanada H, Whiteside LA, Steiger J, et al. Bone landmarks are more reliable than tensioned gaps in TKA component alignment. Clin Orthop Relat Res 2007;462:137-42.

9. Vanin N, Panzica M, Dikos G, et al. Rotational alignment in total knee arthroplasty: intraoperative inter-and intraobserver reliability of Whiteside's line. Arch Orthop Trauma Surg 2011;131:1477-80.

10. Whiteside LA, Arima J. The anteroposterior axis for femoral rotational alignment in valgus total knee arthroplasty. Clin Orthop Relat Res 1995:168-72.

11. Aglietti P, Sensi L, Cuomo P, et al. Rotational position of femoral and tibial components in TKA using the femoral transepicondylar axis. Clin Orthop Relat Res 2008;466:2751-5.

12. Doro LC, Hughes RE, Miller JD, et al. The reproducibility of a kinematically-derived axis of the knee versus digitized anatomical landmarks using a knee navigation system. Open Biomed Eng J 2008;2:52.

13. Lustig S, Lavoie F, Selmi TAS, et al. Relationship between the surgical epicondylar axis and the articular surface of the distal femur: an anatomic study. Knee Surg Sports Traumatol Arthrosc 2008;16:674.

14. Siston RA, Cromie MJ, Gold GE, et al. Averaging different alignment axes improves femoral rotational alignment in computer-navigated total knee arthroplasty. J Bone Joint Surg Am 2008;90:2098-104.

15. Siston RA, Patel JJ, Goodman SB, et al. The variability of femoral rotational alignment in total knee arthroplasty. J Bone Joint Surg Am 2005;87:2276-80.

16. Katz MA, Beck TD, Silber JS, et al. Determining femoral rotational alignment in total knee arthroplasty: reliability of techniques. J Arthroplasty 2001;16:301-5.

17. Mannel H, Marin F, Claes L, et al. Anterior cruciate ligament rupture translates the axes of motion within the knee. Clin Biomech (Bristol, Avon) 2004;19:130-5.

18. Marin F, Sangeux M, Charleux F, et al. Can a finite set of knee extension in supine position be used for a knee functional examination? J Biomech 2006;39:359-63.

19. Colle F, Lopomo N, Visani A, et al. Comparison of three formal methods used to estimate the functional axis of rotation: an extensive in-vivo analysis performed on the knee joint. Comput Methods Biomech Biomed Engin 2016;19:484-92.

20. Kobayashi H, Akamatsu Y, Kumagai K, et al. Is the surgical epicondylar axis the center of rotation in the osteoarthritic knee? J Arthroplasty 2015;30:479-83. 
21. Asano T, Akagi M, Nakamura T. The functional flexionextension axis of the knee corresponds to the surgical epicondylar axis: in vivo analysis using a biplanar imagematching technique. J Arthroplasty 2005;20:1060-7.

22. Colle F, Bignozzi S, Lopomo N, et al. Knee functional flexion axis in osteoarthritic patients: comparison in vivo with transepicondylar axis using a navigation system. Knee Surg Sports Traumatol Arthrosc 2012;20:552-8.

23. Colle F, Bruni D, Iacono F, et al. Changes in the orientation of knee functional flexion axis during passive flexion and extension movements in navigated total knee arthroplasty. Knee Surg Sports Traumatol Arthrosc 2016;24:2461-9.

24. Jerosch J, Peuker E, Philipps B, et al. Interindividual reproducibility in perioperative rotational alignment of femoral components in knee prosthetic surgery using the transepicondylar axis. Knee Surg Sports Traumatol Arthrosc 2002;10:194-7.

25. Yoshino N, Takai S, Ohtsuki Y, et al. Computed tomography measurement of the surgical and clinical transepicondylar axis of the distal femur in osteoarthritic knees. J Arthroplasty 2001;16:493-7.

26. Hirschmann MT, Konala P, Amsler F, et al. The position and orientation of total knee replacement components: a comparison of conventional radiographs, transverse 2DCT slices and 3D-CT reconstruction. J Bone Joint Surg Br 2011;93:629-33.

27. Konigsberg B, Hess R, Hartman C, et al. Inter-and intraobserver reliability of two-dimensional CT scan for total knee arthroplasty component malrotation. Clin Orthop Relat Res 2014;472:212-7.

28. Chambers K, Collings A, Graf C, et al. Towards minimum reporting standards for life scientists. 2019.

29. World Medical Association. World Medical Association Declaration of Helsinki: ethical principles for medical research involving human subjects. JAMA 2013;310:2191-4.

30. Okamoto S, Mizu-uchi H, Okazaki K, et al. Twodimensional planning can result in internal rotation of the femoral component in total knee arthroplasty. Knee Surg Sports Traumatol Arthrosc 2016;24:229-35.

31. Lützner J, Hübel U, Kirschner S, et al. Long-term results in total knee arthroplasty. A meta-analysis of revision rates and functional outcome. Chirurg 2011;82:618-24.

32. Meding JB, Meding LK, Ritter MA, et al. Pain relief and functional improvement remain 20 years after knee arthroplasty. Clin Orthop Relat Res 2012;470:144-9.

33. Wegener JT, van Ooij B, van Dijk CN, et al. Long-term pain and functional disability after total knee arthroplasty with and without single-injection or continuous sciatic nerve block in addition to continuous femoral nerve block: a prospective, 1-year follow-up of a randomized controlled trial. Reg Anesth Pain Med 2013;38:58-63.

34. Wainwright C, Theis J-C, Garneti N, et al. Age at hip or knee joint replacement surgery predicts likelihood of revision surgery. J Bone Joint Surg Br 2011;93:1411-5.

35. Bourne RB, Chesworth BM, Davis AM, et al. Patient satisfaction after total knee arthroplasty: who is satisfied and who is not? Clin Orthop Relat Res 2010;468:57-63.

36. Noble PC, Conditt MA, Cook KF, et al. The John Insall Award: Patient expectations affect satisfaction with total knee arthroplasty. Clin Orthop Relat Res 2006;452:35-43.

37. Parvizi J, Nunley RM, Berend KR, et al. High level of residual symptoms in young patients after total knee arthroplasty. Clin Orthop Relat Res 2014;472:133-7.

38. Matsuda S, Miura H, Nagamine R, et al. Effect of femoral and tibial component position on patellar tracking following total knee arthroplasty: 10-year follow-up of Miller-Galante I knees. Am. J. Knee Surg 2001;14:152-6.

39. Fehring TK. Rotational malalignment of the femoral component in total knee arthroplasty. Clin Orthop Relat Res 2000;380:72-9.

40. Sharma L, Song J, Felson DT, et al. The role of knee alignment in disease progression and functional decline in knee osteoarthritis. JAMA 2001;286:188-95.

41. Griffin FM, Insall JN, Scuderi GR. The posterior condylar angle in osteoarthritic knees. J Arthroplasty 1998;13:812-5.

42. Victor J. Rotational alignment of the distal femur: a literature review. Orthop Traumatol Surg Res 2009;95:365-72.

43. Amaranath JE, Moopanar TR, Sorial RM. Defining distal femoral anatomy for rotational alignment in total knee arthroplasty: a magnetic resonance imaging based study. ANZ J Surg 2014;84:852-5.

44. Incavo SJ, Coughlin KM, Pappas C, et al. Anatomic rotational relationships of the proximal tibia, distal femur, and patella: implications for rotational alignment in total knee arthroplasty. J Arthroplasty 2003;18:643-8.

45. Ritter MA, Davis KE, Meding JB, et al. The effect of alignment and BMI on failure of total knee replacement. J Bone Joint Surg Am 2011;93:1588-96.

46. Kobayashi H, Akamatsu Y, Kumagai K, et al. The surgical epicondylar axis is a consistent reference of the distal femur in the coronal and axial planes. Knee Surg Sports Traumatol Arthrosc 2014;22:2947-53.

47. Jang ES, Connors-Ehlert R, LiArno S, et al. Accuracy of Reference Axes for Femoral Component Rotation in Total 
Knee Arthroplasty: Computed Tomography-Based Study of 2,128 Femora. J Bone Joint Surg Am 2019;101:e125.

48. Wu XD, Xiang BY, Schotanus MGM, et al. CT- versus

Cite this article as: Xiang BY, Wu XD, Zhou N, Li K, Xu W, Liang X, Hu N, Huang W, Qiu GX. Three-dimensional color map: a novel tool to locate the surgical transepicondylar axis. Ann Transl Med 2020;8(21):1401. doi: 10.21037/atm-20-1887
MRI-based patient-specific instrumentation for total knee arthroplasty: A systematic review and meta-analysis. Surgeon 2017;15:336-48. 